\title{
Precipitous neurologic decline following intraventricular rupture of a cerebral abscess: classic imaging findings in ventriculitis and pyocephalus
}

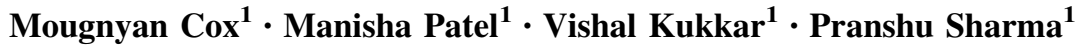

Received: 17 October 2016/Accepted: 22 October 2016/Published online: 27 October 2016

(C) SIMI 2016

\section{Case}

An 80-year-old man was admitted to an outside hospital for a headache, fever, and altered mental status. His admission to neurologic examination was notable for right eye deviation and right gaze preference. Neuroimaging was performed, and showed a ring-enhancing mass with central restricted diffusion in the right occipital lobe consistent with an abscess. During his admission, he became obtunded, and repeat imaging was performed. Repeat contrast-enhanced magnetic resonance imaging showed interval rupture of the right occipital lobe abscess into the adjacent lateral ventricle, with evidence of pyocephalus and ventriculitis. He was transferred to our facility for neurosurgical debridement of his abscess.

Pyogenic ventriculitis is a life-threatening complication of cerebral abscesses caused by rupture and decompression of an abscess into the ventricles [1,2]. Due to differences in blood supply, the wall of a cerebral abscess is usually thinner at its medial margin facing the ventricles, which predisposes rupture at this site. Signs of Intraventricular rupture of an abscess include a direct connection between the abscess and the ventricle, pyogenic debris within the ventricles, and abnormal thickening shown by increased enhancement of the ventricular wall [3]. Diffusion-weighted images are especially helpful, as viscous, pyogenic material is often diffusion-restricting, and will be bright on diffusion-weighted imaging. Delays in detecting ventriculitis can result in death or severe neurologic disability, even after successful treatment of infection [4]. Familiarity

Mougnyan Cox

mougnyan.cox@gmail.com

1 Thomas Jefferson University, 132 South 10th Street, 1087 Main Building, Philadelphia, PA, USA with the common imaging appearance of pyogenic ventriculitis can facilitate earlier diagnosis and treatment of this devastating condition, and potentially improve outcomes.

\section{Figures}

\section{Panel 1}

First image in panel A is a contrast-enhanced brain MRI showing a ring-enhancing abscess (blue arrows) in the right occipital lobe. The second image is a diffusion-weight image, and shows bright internal signal within the abscess, consistent with restricted diffusion. The third image is a Fluid-Attenuated Inversion Recovery sequence (FLAIR), and it shows vasogenic edema surrounding the abscess.

\section{Panel 2}

The first image in panel 2 is a contrast-enhanced sagittal image of the brain showing a connection between the abscess and adjacent right lateral ventricle. The second image is a FLAIR sequence showing bright debris within the dependent portions of the ventricles (compared with the dark signal of normal cerebrospinal fluid within the nondependent portions of both lateral ventricles). On diffusionweighted imaging, the intraventricular debris is diffusionrestricting, consistent with purulent material.

\section{Panel 3}

The blue arrow in this axial contrast-enhanced image of the brain in the same patient as in panels 1 and 2 nicely illustrates abnormal wall thickening and enhancement of the right lateral ventricle compared with the more normal left side. 

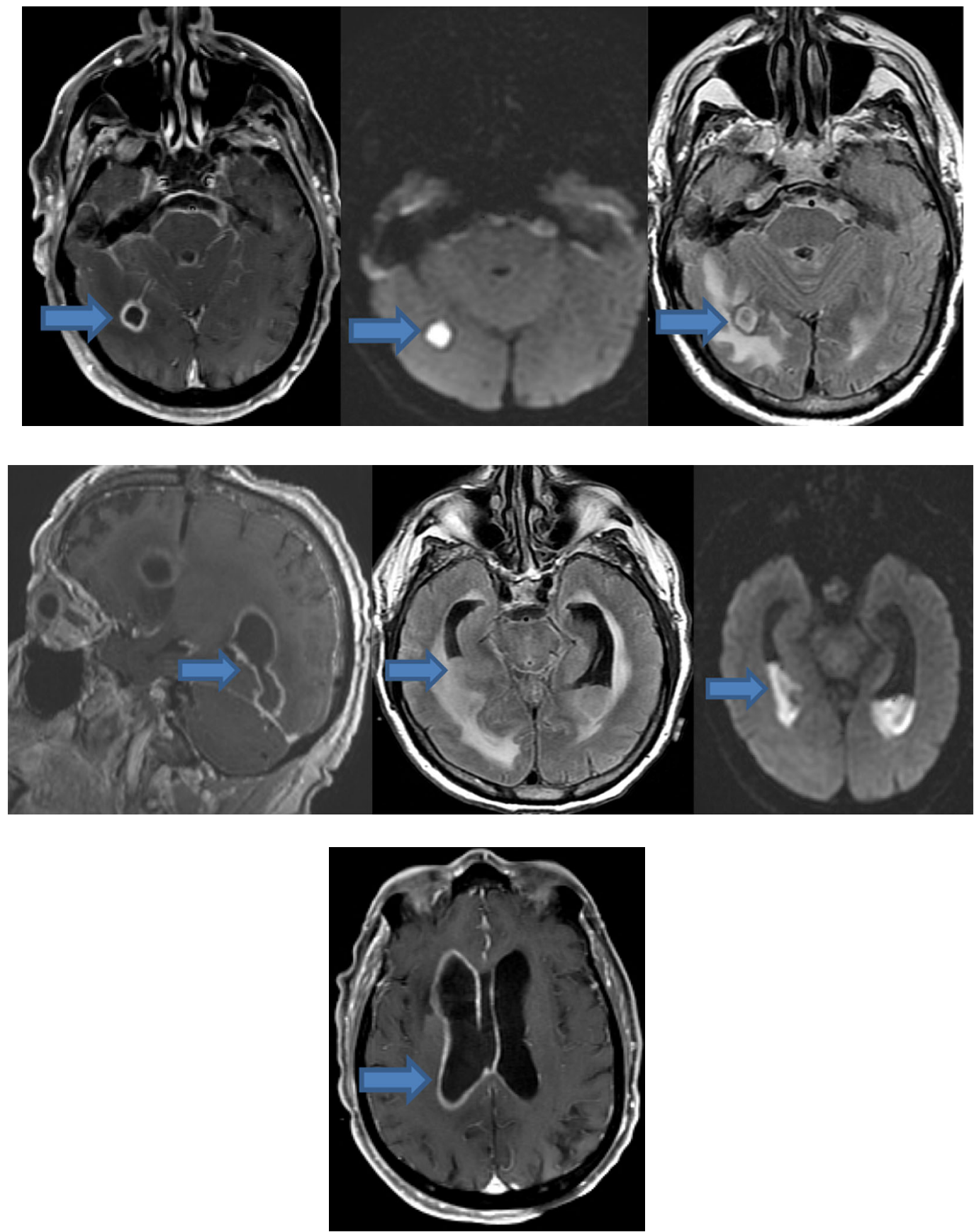

\section{Compliance with ethical standards}

Conflicts of interest The authors declare that they have no conflict of interest.

Statement of human and animal rights All procedures performed in studies involving human participants were in accordance with the ethical standards of the institutional and/or national research committee and with the 1964 Helsinki declaration and its later amendments or comparable ethical standards.

Informed consent Informed consent was obtained prior to all procedures in this study, and all personal identifiers were removed as requested by the institutional review board. 


\section{References}

1. Agrawal A, Cincu R, Timothy J (2008) Current concepts and approach to ventriculitis. Infect Dis Clin Pract 16:100-104

2. Hong JT, Son BC, Sung JH et al (2008) Significance of diffusionweighted imaging and apparent diffusion coefficient maps for the evaluation of pyogenic ventriculitis. Clin Neurol Neurosurg 110:137-144
3. Pezzullo JA, Tung GA, Mudigonda S et al (2003) Diffusionweighted MR imaging of pyogenic ventriculitis. Am J Roentgenol 180:71-75

4. Eholie SP, Boni N, Aoussi E et al (1999) Neurosurgical complications of purulent meningitis in the tropical zone. Neurochirurgie 45:219-224 